\title{
Generalized special functions in the description of fractional diffusive equations
}

\section{Clemente Cesarano ${ }^{1 *}$}

${ }^{1}$ Section of Mathematics, International Telematic University UNINETTUNO, C.so Vittorio Emanuele II 39, 00186 Roma, Italy

*Email address for correspondence: c.cesarano@uninettunouniversity.net

Communicated by Renato Spigler

Received on 11 22, 2018. Accepted on 12 20, 2018.

\section{Abstract}

Starting from the heat equation, we discuss some fractional generalizations of various forms. We propose a method useful for analytic or numerical solutions. By using Hermite polynomials of higher and fractional order, we present some operational techniques to find general solutions of extended form to d'Alembert and Fourier equations. We also show that the solutions of the generalized equations discussed here can be expressed in terms of Hermite-based functions.

Keywords: Hermite polynomials, heat equation, fractional calculus

AMS subject classification: 33 B10, 33 C45, 34 A08, 35 A22

\section{Introduction.}

It is well known that a generalization of the Fourier heat equation for one spatial variable has the form [1]

$$
\left(\frac{\partial}{\partial x}\right)^{m r} u(x, t)-\frac{1}{h}\left(\frac{\partial}{\partial t}\right)^{r} u(x, t)=0
$$

where $m, r \in \mathbb{N}$ and $h \in \mathbb{R}, h \neq 0$.

The integer indices are considered as relatively primes, and $x, t$ variables as complex or real, just like the $h$ constant.

It is also known that the one-dimensional wave equation derives from Eq. 1 when $m=1$ and $r=2$, thus taking the following form:

$$
\left(\frac{\partial}{\partial x}\right)^{2} u(x, t)-\frac{1}{h}\left(\frac{\partial}{\partial t}\right)^{2} u(x, t)=0,
$$

which is known in the literature [2] as the d'Alembert wave equation. Finally, in Eq. 1 one can easily recognize the Fourier heat equation when $m=2$ and $r=1$ :

$$
\left(\frac{\partial}{\partial x}\right)^{2} u(x, t)-\frac{1}{h} \frac{\partial}{\partial t} u(x, t)=0
$$

The simplified (homogeneous) Fourier heat equation [1] is one of the most popular equations in mathematical physics:

$$
\begin{aligned}
\left(\frac{\partial}{\partial x}\right)^{2} u(x, t) & =\frac{\partial}{\partial t} u(x, t) \\
u(x, 0) & =g(x)
\end{aligned}
$$


with $x$ the spacial and $t$ the time variable, usually non-negative.

Its solutions are renowned, and one of its most frequently exploited form is the Gauss transform [3]

$$
u(x, t)=\frac{1}{2 \sqrt{\pi t}} \int_{-\infty}^{+\infty} e^{-\frac{(x-\xi)^{2}}{4 t}} g(\xi) d \xi
$$

which is kind of a convolution of the $g(x)$ function with a Gaussian. Its solution exists when the integral on the r.h.s. of Eq. 5 converges, and for non-negative values of $t$; its fundamental form is

$$
\Phi(x, t)=\frac{1}{2 \sqrt{\pi t}} e^{\left(-\frac{x^{2}}{4 t}\right)}
$$

which is recognized as the Green's function [3], with no limitations on $x$.

A description of the above-mentioned forms of the generalized heat Eq. 1 can be derived by means of a generalized class of Hermite polynomials, as well as some special generalizations of Bessel functions.

\section{Generalized Hermite polynomials and translation operators.}

In order to introduce various classes of Hermite polynomials, one can use a different approach, which exploits the concepts and related formalism of translation operators $[4,5]$.

One can start here from considering a real function, which is analytic in a neighborhood of the origin (but the generalization to the complex case of the properties being discussed is an easy task), so that it can be expanded into a Taylor series. Specifically, one can write

$$
f(x+\lambda)=\sum_{n=0}^{+\infty} f^{(n)}(x)
$$

where $\lambda$ is a continuous parameter.

Under such a hypothesis, a so-called shift (or translation) operator $e^{\lambda \frac{d}{d x}}$ produces a shift of the variable $x$ by parameter $\lambda$ :

$$
e^{\lambda \frac{d}{d x}} f(x)=\sum_{n=0}^{+\infty} \frac{\lambda^{n}}{n !} \frac{d^{n}}{d x^{n}} f(x)=\sum_{n=0}^{+\infty} \frac{\lambda^{n}}{n !} f^{(n)}(x)
$$

which gives, using Eq. 7,

$$
e^{\lambda \frac{d}{d x}} f(x)=f(x+\lambda)
$$

Under the same hypothesis a further relation can be established for $f(x)$, which is the following

$$
e^{\lambda x \frac{d}{d x}}=f\left(e^{\lambda} x\right)
$$

as well as

$$
e^{\lambda x^{2} \frac{d}{d x}}=f\left(\frac{x}{1-\lambda x}\right)
$$

for continuous $\lambda$ 's such that $|x|<\frac{1}{|\lambda|}$. 
There exists a wide theory [4,6] regarding the properties that involve exponential operators; for our purposes, we will focus on some elementary rules, such as the ones mentioned above.

We introduce here the two-variable Hermite polynomials [6] by using the techniques of translation operators. From Eqs. 9, 10, and 11 one can retrieve

$$
e^{y \frac{d^{2}}{d x^{2}}} f(x)=\sum_{n=0}^{+\infty} \frac{y^{n}}{n !} f^{(2 n)}(x),
$$

where again $f(x)$ is an analytic function, and $y$ is a real variable.

By noticing that

$$
D^{2 s} x^{n}=\frac{n !}{(n-2 s) !} x^{n-2 s},
$$

one can derive

$$
e^{y \frac{d^{2}}{d x^{2}}} x^{n}=\sum_{s=0}^{\left[\frac{n}{2}\right]} \frac{y^{s}}{s !} \frac{n !}{(n-2 s) !} x^{n-2 s},
$$

which represents the general action of the exponential operator.

One can now define the two-variable Hermite polynomial of Gould-Hopper type $[7,8]$ by setting the following formula:

$$
H_{n}(x, y)=\sum_{s=0}^{\left[\frac{n}{2}\right]} \frac{n !}{(n-2 s) ! s !} y^{s} x^{n-2 s}
$$

It is important to notice that, assuming

$$
f(x)=\sum_{n=0}^{+\infty} a_{n} x^{n},
$$

one can immediately obtain the following identity:

$$
e^{y \frac{d^{2}}{d x^{2}}} f(x)=\sum_{n=0}^{+\infty} a_{n} H_{n}(x, y) .
$$

From the above relations, one can derive an elementary form of this kind of Hermite polynomials. Indeed, from identity 12 one infers:

$$
H_{n}^{(1)}(x, y)=(x+y)^{n},
$$

where $H_{n}^{(1)}(x, y)$ is a slight variant of $H_{n}(x, y)$.

The two-variable Hermite polynomials of type $H_{n}(x, y)$ are linked to the ordinary Hermite polynomials by the following relation [5]:

$$
H_{n}\left(x,-\frac{1}{2}\right)=H_{e_{n}}(x)
$$




\section{Cesarano}

where:

$$
H_{e_{n}}(x)=\sum_{s=0}^{\left[\frac{n}{2}\right]} \frac{n !(-1)^{s} x^{n-2 s}}{(n-2 s) ! s ! 2^{s}} .
$$

It must be stressed that the definition of the Hermite polynomials $H_{n}(x, y)$ is not limited to positive values of $y$, so that, in principle, one can use this family of polynomials as a basis to get solutions for generalized heat equations.

It is useful to notice that the $H_{n}(x, y)$ polynomials solve the following partial differential equation $[5,9]$ :

$$
\frac{\partial}{\partial y} H_{n}(x, y)=\frac{\partial^{2}}{\partial x^{2}} H_{n}(x, y)
$$

which can be easily recognized as belonging to the general case of generalized heat equations presented in the previous section (see Eqs. 1 and 4).

In order to prove relation 21 , one can notice that, by separate differentiation with respect to $x$ and $y$ in Eq. 15, the following relations are derived:

$$
\begin{aligned}
\frac{\partial}{\partial x} H_{n}(x, y) & =n H_{n-1}(x, y) \\
\frac{\partial}{\partial y} H_{n}(x, y) & =n(n-1) H_{n-2}(x, y)
\end{aligned}
$$

furthermore, differentiating the former equation in cluster 22 with respect to $x$, the relation

$$
\frac{\partial^{2}}{\partial x^{2}} H_{n}(x, y)=n(n-1) H_{n-2}(x, y)
$$

is retrieved, which, combined to the latter, allows for concluding that Eq. 21 holds true.

It is worth noticing that, starting from the explicit form of two-variable Hermite polynomials (see Eq. 15), one can explore a special case:

$$
H_{n}(x, y=0)=x^{n} .
$$

Eq. 24 suggests one to read Eq. 21 as ordinary in the $y$ variable; Eq. 21 is also linear, so that one can consider the following Cauchy problem:

$$
\begin{aligned}
\frac{\partial}{\partial y} H_{n}(x, y) & =\frac{\partial^{2}}{\partial x^{2}} H_{n}(x, y) \\
H_{n}(x, 0) & =x^{n},
\end{aligned}
$$

and straightforwardly get the solution $[5,9,10]$ :

$$
H_{n}(x, y)=e^{y \frac{\partial^{2}}{\partial x^{2}}} x^{n}
$$

which can also be interpreted as a proof of our early definition of Hermite polynomials of type $H_{n}(x, y)$ given in Eq. 14.

We will use the formalism presented in this section to find solutions of generalized d'Alembert and Fourier equations presented in the Introduction. 


\section{Solutions of generalized d'Alembert and Fourier equations.}

Starting from Eq. 1, we consider here the case $m=1$ and $r$ any positive integer:

$$
\left(\frac{\partial}{\partial x}\right)^{r} x(x, t)-\frac{1}{h}\left(\frac{\partial}{\partial t}\right)^{r} u(x, t)=0
$$

In order to write the general solution, we start from the case $r=1$, and notice that Eq. 27 can be formally rewritten as

$$
\frac{\partial}{\partial t} u(x, t)=h \hat{\Theta} u(x, t)
$$

where $\hat{\Theta}=\frac{\partial}{\partial x}$.

Clearly, operator $\hat{\Theta}$ can be treated as a constant, thus Eq. 28 can be read as an ordinary, linear differential equation with respect to $y$, by setting the initial condition

$$
u(x, 0)=g(x)
$$

with $g(x)$ analytic in a neighborhood of the origin, that is,

$$
g(x)=\sum_{n=0}^{+\infty} a_{n} x^{n},
$$

one can immediately state the solution of the Cauchy problem linked to Eq. 28:

$$
u(x, t)=e^{h t \hat{\Theta}}[g(x)],
$$

i.e.,

$$
u(x, t)=e^{h t \frac{\partial}{\partial x}}[g(x)] .
$$

Hence, using properties of exponential operators discussed in the previous Section (Eq. 9 in particular), one obtains

$$
u(x, t)=g(x+h t),
$$

which provides the most general solution of the partial differential equation appearing in Eq. 1 for the case $m=1, r \geq 1$.

It is worth noticing here that the classical d'Alembert solution of the wave propagation is obtaned by setting $r=2$.

Furthermore, we have proven that two-variable Hermite polynomials of type $H_{n}(x, y)$ solve the Cauchy problem appearing in Eq. 25, from which Eq. 26 was deduced.

That said, remembering that the generalized heat equation (Eq. 1) for $m=2$ and $r=1$ can be recognized as the Fourier heat equation (see Eq. 4),

one gets the solution

$$
u(x, t)=e^{h t\left(\frac{\partial}{\partial x}\right)^{2}}[g(x)] .
$$


In addition, using Eq. 17, one can recast the above expression in a more convenient form. Indeed, many families of Hermite polynomials can be considered quasi-monomials [11] under the action of particular operators and the setting of specific hypotheses. As to Hermite polynomials, it is common knowledge [12] that they are quasi-monomial with respect to the operators

$$
\hat{M}=x+2 y \frac{\partial}{\partial x}, \quad \hat{P}=\frac{\partial}{\partial x}
$$

with rules

$$
\begin{aligned}
\hat{M} H_{n}(x, y) & =H_{n+1}(x, y) \\
\hat{P} H_{n}(x, y) & =n H_{n-1}(x, y)
\end{aligned}
$$

holding true.

In this context, it is possible to introduce many families of hybrid polynomials [13] and generalized special functions [14], in the sense that one can replace the monomial variable with a quasi-monomial one, which, in our discussion, is the Hermite polynomial of type $H_{n}(x, y)$.

Taking into account the above, one can define, for an analytic real function $g(x)$, its generalization based on the $H_{n}(x, y)$ polynomials, that is,

$$
{ }_{H} g(x, y)=\sum_{n=0}^{+\infty} a_{n} H_{n}(x, y) .
$$

Finally, observing the structure of Eq. 34 and using Eq. 17, one can state a further, explicit form of the solution for the generalized heat equation in the case $m=2, r=1$, that is,

$$
u(x, t)={ }_{H} g(x, h t),
$$

where

$$
{ }_{H} g(x, h t)=\sum_{n=0}^{+\infty} a_{n} H_{n}(x, h t) .
$$

A different method to generalize, and consequently to solve, equations belonging to the class of the heat equation, consists in directly working on the Hermite polynomials to derive generalized families, then retrieving a different form of heat equations from these latter families.

To this aim, one introduces the Hermite polynomials of type $H_{n}^{(m)}(x, y)$ by setting [9]:

$$
H_{n}^{(m)}(x, y)=\sum_{s=0}^{\left[\frac{n}{m}\right]} \frac{n !}{s !(n-m s) !} y^{s} x^{n-m s}
$$

with $m \in \mathbb{N}$.

It is easy to realize that the above expression comes from the concepts, and the related formalism, of exponential operators presented in Section 2. Indeed, starting from Eq. 12, one can write:

$$
e^{y \frac{d^{m}}{d x^{m}}} f(x)=\sum_{n=0}^{+\infty} \frac{y^{n}}{n !} f^{(n m)}(x)
$$


and, since

$$
\frac{d^{m s}}{d x^{m s}} x^{n}=n(n-1) \cdots(n-m s+1) x^{n-m s}=\frac{n !}{(n-m s) !} x^{n-m s}
$$

for $s=0,1, \ldots,\left[\frac{n}{m}\right]$, then one gets

$$
e^{y \frac{d^{m}}{d x^{m}}} x^{n}=\sum_{n=0}^{\left[\frac{n}{m}\right]} \frac{y^{s}}{s !} \frac{n !}{(n-m s) !} x^{(n-m s)} .
$$

In order to prove the expression appearing in Eq. 40, it is interesting to notice that the Hermite polynomials $H_{n}^{(m)}(x, y)$ can be equivalently introduced by direct use of their generating function. Indeed, exploiting the exponential $e^{x t+y t^{m}}$, the identity

$$
e^{x t+y t^{m}}=\sum_{n=0}^{+\infty} \frac{t^{n}}{n !} H_{n}^{(m)}(x, y)
$$

is immediately retrieved, which, when $m=2$, returns the generating function of the Hermite polynomials $H_{n}(x, y)$ :

$$
e^{x t+y t^{2}}=\sum_{n=0}^{+\infty} \frac{t^{n}}{n !} H_{n}(x, y) .
$$

Following the same procedure used for polynomials $H_{n}(x, y)$, one can derive relevant differential properties for the class of Hermite polynomials named $H_{n}^{(m)}(x, y)$.

By differentiation with respect to $y$ in Eq. 44, one recovers:

$$
\sum_{n=0}^{+\infty} \frac{t^{n+m}}{n !} H_{n}^{(m)}(x, y)=\sum_{n=0}^{+\infty} \frac{t^{n}}{n !} \frac{\partial}{\partial y} H_{n}^{(m)}(x, y),
$$

and, after manipulation of the l.h.s., and pairing of $t$-powers of the same order, in Eq. 46, one can write:

$$
\frac{n !}{(n-m) !} H_{n-m}^{(m)}(x, y)=\frac{\partial}{\partial y} H_{n}^{(m)}(x, y)
$$

On the other hand, differentiating Eq. $44 \mathrm{~m}$ times with respect to $x$, one derives:

$$
\begin{aligned}
\frac{\partial}{\partial x} H_{n}^{(m)}(x, y) & =n H_{n-1}^{(m)}(x, y) \\
\frac{\partial^{m}}{\partial x^{m}} H_{n}^{(m)}(x, y) & =\frac{n !}{(n-m) !} H_{n-m}^{(m)}(x, y) .
\end{aligned}
$$

By comparison of Eqs. 47 and 48, one desumes that the Hermite polynomials of type $H_{n}^{(m)}(x, y)$ satisfy the following partial differential equation $[5,10]$ :

$$
\frac{\partial}{\partial y} H_{n}^{(m)}(x, y)=\frac{\partial^{m}}{\partial x^{m}} H_{n}^{(m)}(x, y)
$$


On the other hand, for $y=0$ the polynomials $H_{n}^{(m)}(x, y)$ return:

$$
H_{n}^{(m)}(x, 0)=x^{n}
$$

Then, one can observe that Eq. 49 is ordinary and linear in the $y$ variable, thus recovering the solution of the Cauchy problem with initial condition $y=0$ :

$$
H_{n}^{(m)}(x, y)=e^{y \frac{\partial^{m}}{\partial x^{m}}} x^{n} .
$$

It is evident that Eq. 49 is a slighty generalized form of the heat equation presented in the Introduction. Taking Eq. 1 into account, this suggests that a natural generalization can be implemented on an analytic function $g(x)$ in order to introduce an $m^{\text {th }}$-order Hermite-based function by setting $[15,16]$ :

$$
e^{y \frac{\partial^{m}}{\partial x^{m}}} g(x)={ }_{H^{(m)}} g(x, y)=\sum_{n=0}^{+\infty} a_{n} H_{n}^{(m)}(x, y) .
$$

This last retrieval allows for obtaining the solution for the general case of the formal heat Eq. 1 . Indeed, rewriting Eq. 1 in the form

$$
\left(\frac{\partial}{\partial t}\right)^{r} u(x, t)=h\left(\frac{\partial}{\partial x}\right)^{m r} u(x, t)
$$

for any $m \in \mathbb{N}$, one is left with

$$
u(x, t)=e^{h t \frac{\partial^{m}}{\partial x^{m}}}[g(x)] .
$$

After setting $u(x, 0)=g(x)$, under the same hypothesis given above, and taking into account Eqs. 52 , one gets:

$$
u(x, t)={ }_{H^{(m)}} g(x, h t)=\sum_{n=0}^{+\infty} a_{n} H_{n}^{(m)}(x, h t),
$$

which represents the general solution of the heat equation 53 .

\section{Concluding remarks.}

Before concluding, it is useful to emphasize the clear flexibility and potential of the classes of Hermite polynomials used here to find the solutions of some generalization of the heat equation [17].

One can consider a generalized form of the Fourier heat equation, namely

$$
\left(\frac{\partial}{\partial t}\right) u(x, t)=\left(\frac{\partial}{\partial x}\right)^{\mu} u(x, t)
$$

where the differentiation order is any real value such that $0<\mu<1$, from which one can describe Eq. 56 as a fractional diffusive equation.

In order to treat a sort of fractional-order, generalized heat equation as displayed in Eq. 56, one can use an approach based on a further generalization of Hermite polynomials. 
First, one can introduce, without any restriction, the following object $[9,17]$ :

$$
H_{n}^{(\mu)}(x, y)=\sum_{s=0}^{\left[\frac{n+\mu}{\mu}\right]} \frac{n !}{s ! \Gamma(n-\mu s+1)} y^{s} x^{n-\mu s},
$$

which can be considered as the fractional order counterpart of the $m^{t h}$-order Hermite polynomials appearing in Eq. 40.

Strictly speaking, $H_{n}^{(\mu)}(x, y)$ are functions rather than polynomial functions. It is possible to prove that such functions, defined in Eq. 57, satisfy all the formal properties of the Hermite polynomials of type $H_{n}^{(m)}(x, y)$; and, in particular, that these Hermite fractional functions solve the following partial differential equations:

$$
\frac{\partial}{\partial y} H_{n}^{(\mu)}(x, y)=\frac{\partial^{\mu}}{\partial x^{\mu}} H_{n}^{(\mu)}(x, y)
$$

Since it is also verified that

$$
H_{n}^{(\mu)}(x, 0)=x^{n}
$$

one can conclude that these functions are a basis to derive the solution of a fractional diffusive equation, namely

$$
\mu(x, t)=\sum_{n=0}^{+\infty} a_{n} H_{n}^{(\mu)}(x, y) .
$$

A first benchmark has been attempted of the correctness of the procedure by assessment of the method actually providing the solution

$$
\mu(x, t)=g(x+t)
$$

in the limit $\mu \rightarrow 1$.

This single check cannot be considered sufficient to assess the validity ot the procedure; indeed, a comparison with an independent method must be discussed, based on an integral transform technique.

Specifically, since it is possible to represent generalized two-variable Bessel functions in terms of some special class of Hermite polynomials, one can also investigate how obtaining different expressions of the above results by using special families of Bessel functions.

\section{References}

1. R. Haberman, Applied partial differential equations with Fourier series and boundary value problems. Pearson Higher Ed, 2012.

2. L. C. Evans, Partial differential equations (Providence, ri: American Mathematical Society), 1998.

3. M. Abramowitz and I. A. Stegun, Handbook of mathematical functions: with formulas, graphs, and mathematical tables, vol. 55. Courier Corporation, 1965.

4. B. M. Levitan, Generalized translation operators and some of their applications, 1964.

5. C. Cesarano, G. M. Cennamo, and L. Placidi, Operational methods for Hermite polynomials with applications, WSEAS Transactions on Mathematics, vol. 13, pp. 925-931, 2014. 
6. W. Miller, Lie theory and special functions. Academic Press, 1968.

7. H. W. Gould, A. Hopper, et al., Operational formulas connected with two generalizations of Hermite polynomials, Duke Mathematical Journal, vol. 29, no. 1, pp. 51-63, 1962.

8. P. Appell and J. K. de Fériet, Fonctions Hypergéométriques et Hypersphériques: Polynômes d'Hermite. Paris: Gauthier-Villars, 1926.

9. C. Cesarano, Operational methods and new identities for Hermite polynomials, Mathematical Modelling of Natural Phenomena, vol. 12, no. 3, pp. 44-50, 2017.

10. C. Cesarano, C. Fornaro, and L. Vazquez, Operational results in bi-orthogonal Hermite functions, Acta Mathematica Universitatis Comenianae, vol. 85, no. 1, pp. 43-68, 2016.

11. C. Cesarano, C. Fornaro, and L. Vazquez, A note on a special class of Hermite polynomials, International Journal of Pure and Applied Mathematics, vol. 98, no. 2, pp. 261-273, 2015.

12. H. M. Srivastava and Y. B. Cheikh, Orthogonality of some polynomial sets via quasi-monomiality, Applied Mathematics and Computation, vol. 141, no. 2-3, pp. 415-425, 2003.

13. G. Dattoli*, S. Lorenzutta, P. Ricci, and C. Cesarano, On a family of hybrid polynomials, Integral Transforms and Special Functions, vol. 15, no. 6, pp. 485-490, 2004.

14. C. Cesarano and D. Assante, A note on generalized Bessel functions, International Journal of Mathematical Models and Methods in Applied Sciences, vol. 7, no. 6, pp. 625-629, 2013.

15. C. Cesarano, B. Germano, and P. Ricci, Laguerre-type Bessel functions, Integral transforms and special functions, vol. 16, no. 4, pp. 315-322, 2005.

16. C. Cesarano and P. Ricci, The legendre polynomials as a basis for Bessel functions, International Journal of Pure and Applied Mathematics, vol. 111, no. 1, pp. 129-139, 2016.

17. D. Assante, C. Cesarano, C. Fornaro, and L. Vazquez, Higher order and fractional diffusive equations, Journal of Engineering Science and Technology Review, vol. 8, no. 5, pp. 202-204, 2015. 